\title{
Dengue threat lurks among COVID-19 pandemic
}

(BIRDEM Med J 2020; 10, COVID Supplement: 4-5)

\section{INTRODUCTION}

Communicable viral diseases like dengue, flu and corona are still life threatening through months, years, decades and centuries. Dengue is prevalent in our country since 2000 . We have been habituated with dengue with our accomplished knowledge and experience. We are in evolution of knowledge about the devastating corona. COVID-19 is pandemic, dengue is endemic in warmer regions of the globe. Treatments of both are supportive and symptomatic. Most of the cases are simple infection and can be fatal and panic to mankind. So far, we faced several viral epidemics; swine flu by (Pandemic flu 2009) H1N1, COVID-19 by SARS-CoV-2, SARS, MERS corona by SARS-CoV-1, dengue by DEN 1 to 4 and chikungunya by Chik-V.

\section{Transmission}

All flu are transmitted by droplet, fomite and transmission is from human to human. Dengue and chikugunya are transmitted by mosquitoes bites. All flu remained static (one by one) but for COVID-19 it is exponential... 82 percent of population will have to be immune (either by disease or by vaccine) to stop transmission of this disease (herd immunity). Human transmission is not possible in dengue. Dengue is basically a seasonal disease occurring mainly in rainy season. It is said that dengue like other vector-borne diseases is the outcome of the effect of global warming. We have witnessed first outbreak in 2000. So far, we have seen maximum incidence is in the month of September and October. This year, even in November, we are getting dengue cases.

\section{Pathophysiology}

In about 80 percent of COVID-19 patients, innate and adaptive immune system work perfectly and patients recover either with mild or moderate symptoms. Natural killer (NK) cells and cytotoxic T lymphocytes (CTLs) clear virally affected cells, damaged cells and give a negative feedback to macrophage. In about 20 percent cases, immune system fails to work properly and we see the severe disease and critical complications. In these groups of patients, antibody response is inadequate; NK cells and CTLs fail to clear the damaged cells and fail to inhibit macrophage activity. In severe and critical diseases, there is cytokine release syndrome (CRS)/ macrophage activation syndrome/hemophagocytic lymphohistiocytosis. There is hypercoagulable state and widespread thrombo-embolic complication of both pulmonary and systemic circulation. In dengue, the neutralizing antibody helps combat first infection and protect for one year but antibody enhanced immune response cause the severe disease in subsequent infection by different strain. One infection by a strain gives life-long immunity to that strain. Dengue predominantly affects the homeostasis of the body through leaking of the blood vessels and bleeding manifestation. There is endothelial dysfunction due to enhance viral activity, cytokines, autoimmunity and $\mathrm{T}$ cell apoptosis. More the virus more is macrophage activities, more is the damage.

\section{Clinical features}

In flu and dengue, initial manifestation is indistinguishable; fever, malaise, cough. Main target of involvement in COVID-19 is respiratory system while dengue causes blood vessel leaking. All the viral diseases are multisystem affecting disorder each have characteristic skin manifestation.

Category 1. Mild illness: In COVID-19, it is influenza like illness (ILI) and pneumonia [CURB $<2$ while in dengue it is classical dengue.

Category 2. Moderate: In flu it is severe acute respiratory illness (SARI) requiring oxygen and in dengue, it is dengue with warning sign requiring IV fluid; both groups may need hospitalization. Category 3. Severe: Severe form of dengue viz dengue shock syndrome (DSS) and is characterized by severe plasma leakage, severe internal bleeding, severe organ failure (ALT $>1000 \mathrm{U} / \mathrm{L}$ ), acute kidney injury (AKI), cardiomyopathy, encephalitis and multi-organ failure. COVID-19, in its severe form: oxygen saturation $<93 \%$ on room air, $\mathrm{Pao} 2 / \mathrm{Fio} 2<300 \mathrm{mmHg}$, respiratory rate 
$>25$ breaths/minute or respiratory distress with $>50 \%$ involvement of lung parenchyma on radiology. Twenty percent of COVID-19 cases become severe. Both diseases need hospitalizations in high dependency units (HDU)/intensive care units (ICU)...5\% of COVID-19 pneumonia needs ICU.

\section{Special forms}

1. Vulnerable peoples: elderly people and patients with pregnancy and with co-morbidities like renal, hepatic, pulmonary and cardiac diseases.

2. Atypical form: virus can affect any organ, may have superadded infection, may induce inflammation. So, (a) COVID-19 may present with stroke/ thromboembolism/myocarditis (b) dengue with encephalitis/cardiomyopathy.

3. Sequalae a) dengue expanded syndrome, PUO, chronic fatigue syndrome; b) COVID-19 with aspergillous pneumonia and long COVID.

\section{Special groups}

1. Pregnancy: COVID-19, complication of the disease like SARI and consequences are more. In dengue, pre-eclamsia needs to be excluded. It is not an indication of termination of pregnancy or early Cesarean section. Fetal complications are reported. Vertical transmission is possible in both groups. Fetal screening is indicated in dengue. Mandatory similar individual safety measures advocated are for both diseases.
2. Breast feeding is not contraindicated, mother should take necessary precaution.

\section{Peculiarities}

In COVID-19: If SPO2 can be maintained with oxygen to $>95 \%$, victory over corona is possible, $\mathrm{D}$-dimer is a marker of severity. Intravascular micro-thrombi and not DIC determines outcome. In dengue: Fluid (crystalloids) and its judicial use is the treatment. Platelet is a marker of severity, DIC determine outcome.

Confirmatory/screening test: RT-PCR for corona with nasopharyngeal or throat swab confirms SARS-CoV-2 with $30 \%$ false negativity; it is not done in dengue. Viral culture is only done in dengue for research purposes blood sample to be taken in febrile period.

Antigen: NS1 antigen helps in first half of febrile phase. Antibody tests are not done in our country in COVID19, which helps in surveillances and becomes positive 7 days onwards.

\section{CONCLUSION}

Communicable viral diseases like dengue and COVID19 is prevalent in our country. These diseases have disrupted our lives in every aspect. Individual's safety is on his own hand. Universal face masking, herd immunity, effective vaccine remain the cornerstone of COVID prevention. Prevalence of dengue can be reduced with effective mosquito control. 